\title{
Correction: Dimethyl fumarate for psoriasis
}

Our article Dimethyl fumarate for psoriasis (DTB 2017; 55: 141-4) contained three numerical errors. In the Cost section, the maximum dose should have read "720mg daily". In the Conclusion, the first sentence of the second paragraph should have read "Dimethyl fumarate produces a clinically significant improvement in psoriasis in around 38\% of patients who take it (compared with 15\% with placebo) after 16 weeks." These changes do not alter our overall conclusion. 